\title{
КВАНТОВАЯ ЭЛЕКТРОНИКА
}

\section{В.П. ЦИПИЛЕВ, В.И. ОЛЕШКО, А.Н. ЯКОВЛЕВ, Н.А. АЛЕКСЕЕВ, О.В. НОЗДРИНА, М.А. МАЗУР}

\section{НАУЧНО-МЕТОДИЧЕСКИЕ ПОДХОДЫ ИЗМЕРЕНИЯ ХАРАКТЕРИСТИК ФИЗИКО-ХИМИЧЕСКИХ ПРОЦЕССОВ В КОНДЕНСИРОВАННЫХ СРЕДАХ ПРИ ВОЗДЕЙСТВИИ ЛАЗЕРНОГО ИЗЛУЧЕНИЯ И ИХ ЭКСПЕРИМЕНТАЛЬНАЯ РЕАЛИЗАЦИЯ}

\begin{abstract}
Представлены научно-методические подходы и созданная экспериментальная установка, предназначенная для исследования нелинейных физико-химических процессов, сопровождающих воздействие лазеров, работающих в различных областях спектра (УФ, видимая и ИК) на твердые тела различных классов, в том числе на энергетические материалы. Продемонстрирована возможность проводить синхронные многопараметрические измерения с наносекундным временным разрешением амплитудных, спектральных, кинетических и пространственных характеристик свечения приповерхностной и объемной лазерной плазмы, люминесценции твердой фазы, акустических импульсов, возникающих в объеме образцов и морфологии остаточных разрушений. Плотность энергии на поверхности облучаемых мишеней в зависимости от задачи варьировалась от долей мДж/см² до $10^{4}$ Дж/см². Спектральный интервал, регистрируемый за один импульс облучения, составлял 200-1100 нм, спектральное разрешение $\sim 1.5$ нм, пространственное $\sim 10$ мкм. Импульс давления, возникающий в объеме образцов, регистрировался акустическим датчиком с чувствительностью 0.15 В/бар и временным разрешением $\sim 5$ нс.
\end{abstract}

Ключевые слова: лазерное воздействие, инертные и энергетические материалы, лазерная плазма, горячие точки, взрывное свечение, спектроскопия, оптоакустика.

\section{Введение}

В течение последних десятилетий проводятся фундаментальные исследования свойств вещества в экстремальных условиях. Большой объем работ включает изучение нелинейных физикохимических процессов, инициируемых мощными лазерными пучками в различных материалах [114]. Широкие возможности для анализа эксперимента и теории дают многопараметрические измерения амплитудных, спектральных, кинетических и пространственных характеристик свечения, акустических импульсов, морфологии остаточных разрушений и оптических свойств поверхности, регистрируемых в момент и после импульса облучения образцов. Особенно актуальны такие исследования при изучении механизмов взрывного разложения энергетических материалов (ЭМ), в том числе процессов и явлений, предшествующих взрыву. Ранее проведенные исследования показали большие возможности синхронных многопараметрических измерений с высоким временным разрешением для получения информации о первичных процессах, развивающихся во взрывчатых веществах под действием лазерных пучков [15-20]. Однако возникают проблемы физического понимания результатов экспериментов, что затрудняет выработку общепринятых представлений о механизмах тех или иных явлений, индуцированных лазерным импульсом в материалах. В частности, широко дискутируется вопрос о механизмах лазерного инициирования ЭМ. Эти исследования выявили научные и методические трудности, связанные со спецификой взаимодействия мощного лазерного излучения с твердыми телами различных классов. К настоящему времени стало ясно, что при исследовании процессов инициирования взрывчатых веществ необходимо выполнение ряда условий, без соблюдения которых их интерпретация вызывает сомнения. Во-первых, необходимо, чтобы эксперименты проводились при воздействии лазерным пучком с однородным распределением освещенности по поперечному сечению и с резким контрастом на его границах. Вовторых, эксперименты требуют измерения спектров и кинетики различных видов свечений с высоким спектральным, временным и пространственным разрешением, в том числе за один импульс возбуждения. Такие измерения позволяют выделить процессы, протекающие в твердом теле, от процессов, развивающихся в приповерхностной лазерной плазме. В-третьих, необходимо обеспечить возможность изучения поведения материала в широком диапазоне длин волн, длительностей импульсов, размеров лазерного пучка, что позволит определить пространственно-временные мас-

\footnotetext{
* Работа выполнена в рамках Программы повышения конкурентоспособности ТПУ.
} 
Уважаемые читатели!

Доступ к полнотекстовой версии журнала
«Известия высших учебных заведений. Физика» осуществляется на платформе Научной электронной библиотеки eLIBRARY.RU на платной основе:

https://elibrary.ru/contents.asp?titleid=7725 